\title{
Influence of sintering temperature on the structural, electrical and microwave properties of yttrium iron garnet (YIG)
}

\begin{abstract}
This study investigates the structural, electrical and microwave properties of yttrium iron garnet (YIG) which focuses on the parallel evolving relationship with their dependence on the sintering temperature. The iron oxide obtained from the steel waste product (mill scale) was used to synthesize YIG. The raw mill scale underwent the milling and Curie temperature separation technique to produce high purity iron oxide powder which is the main raw material in preparing and fabricating YIG through high energy ball milling (HEBM) process. Microstructural features such as amorphous phase, grain boundary, secondary phase and intergranular pores contribute significantly to the additional magnetic anisotropy and demagnetizing fields, affecting the electric and microwave properties accordingly. The increment in electrical resistivity and decrement in linewidth while the microstructure was evolving is believed to be a strong indicator of improved phase purity and compositional stoichiometry.
\end{abstract}

Keyword: Yttrium iron garnet; Yttrium compounds; Microstructural evolution; Microstructural features; Sintering temperatures 